\title{
Inverse Heat Conduction Problems by Using Particular Solutions
}

\author{
P.H. Wen, ${ }^{1}$ Y.C. Hon, ${ }^{2}$ and Y.G. $\mathrm{Xu}^{3}$ \\ ${ }^{1}$ School of Engineering and Materials Science, Queen Mary, University of London, \\ London, UK \\ ${ }^{2}$ Department of Mathematics, City University of Hong Kong, Hong Kong SAR, China \\ ${ }^{3}$ School of Engineering and Technology, University of Hertfordshire, Hatfield, Herts, UK
}

\begin{abstract}
Based on the method of fundamental solutions, we develop in this paper a new computational method to solve two-dimensional transient heat conduction inverse problems. The main idea is to use particular solutions as radial basis functions (PSRBF) for approximation of the solutions to the inverse heat conduction problems. The heat conduction equations are first analyzed in the Laplace transformed domain and the Durbin inversion method is then used to determine the solutions in the time domain. Least-square and singular value decomposition (SVD) techniques are adopted to solve the ill-conditioned linear system of algebraic equations obtained from the proposed PSRBF method. To demonstrate the effectiveness and simplicity of this approach, several numerical examples are given with satisfactory accuracy and stability.
\end{abstract}

Key words: transient inverse heat conduction problem, Laplace transform, Durbin algorithm, singular value decomposition, meshless collocation method

\section{Introduction}

Inverse heat conduction problems (IHCP) arise from the studies in many fields of science and engineering such as modeling and control of processes with heat propagation in thermo-physics and mechanics of continuous media. A typical inverse heat conduction problem is to compute the unknown temperature and heat flux at an unreachable boundary from scattered temperature measurements at reachable interior points or boundary of the domain. In practice, some kinds of non-destructive evaluation techniques to determine the interior heat flows in an inaccessible domain from exterior scattered data are indispensable for industrial and engineering applications. It is well known that inverse problems are by nature 'unstable' in the sense that small measurement errors in the input data may amplify significantly the errors in the solutions. Most traditional computational methods including the finite element method and boundary element method for well-posed direct problems

Contract grant sponsor: Research Grant Council of the Hong Kong Special Administrative Region, China (Project No. CityU 101209). 
fail to produce acceptable solutions to these kinds of inverse problems. Several techniques have been proposed for solving a one-dimensional IHCP [1-5]. Among the methods proposed for higher dimensional IHCP, boundary element [6, 7], finite difference [8,9], and finite elements $[10,11]$ have successfully been developed for two-dimensional IHCP and, more recently, the method of fundamental solutions [12] for problems in three dimensions.

Despite the great success of the finite and boundary element methods as effective numerical tools for the solution of boundary value problems, there is still a need for developing numerical schemes for multi-dimensional IHCP. The meshless methods are becoming popular due to their high adaptability and low cost to prepare input data for numerical analyses. Various meshless methods, such as the method of fundamental solutions (MFS) [13-15], meshless local Petrov-Galerkin method $[16,17]$, and element-free Galerkin method [18], have recently attracted great attention in science and engineering communities for numerically solving time-dependent problems. The MFS is a boundarytype meshless strong collocation method which is highly accurate for solving homogeneous equations if the fundamental solution of the given differential operator is known [13, 14]. The fundamental solution of a given differential equation, however, is not always available and often very difficult to derive. The ill-conditioning of the resultant matrix and the optimal placement of source points in using the MFS are still open research problems. To extend the MFS to solve inhomogeneous equations or time-dependent problems, some boundary-type meshless methods [19-21] have been introduced to evaluate the particular solution of the given differential equation. Since the particular solution is not unique, there is a rich variety of numerical techniques developed for this purpose. For instance, radial basis functions, polynomial functions, and trigonometric functions have been employed in Refs. 13 and 21 as the basis functions to approximate the particular solutions of the given partial differential equations.

In the last decade, the radial basis functions (RBF) have been under intensive research in the areas of multivariate function interpolation and partial differential equations solver [16, 18, 22]. A new non-iterative numerical method for solving inverse boundary value problems for linear elliptic equations of second order was presented by J. Li [23]. In this paper we propose an alternative method PSRBF for solving the inverse heat conduction problems using the particular or semi-particular solutions as radial basis functions. Applying the newly developed PSRBF, we can solve the elliptic equation in the Laplace domain. The solution of the IHCP is then obtained by using the Durbin inversion method. To solve the ill-conditioned linear system of algebraic equations obtained from the PRBS, boundary conditions and scattered measurement data of heat either on the boundary or in the domain, we adopt the use of the least-square method (LSM) and singular value decomposition (SVD) $[24,25]$. For numerical verification, we solve a series of boundary value problems for various values of the Laplace transform parameter. Subsequently, the Durbin inversion method [26] is applied to obtain the solution in the time domain. Three numerical examples are given to demonstrate the effectiveness and stability of the proposed approach in comparison with analytical solutions.

\section{Formulation of Inverse Heat Conduction Problems}

Consider the following linear initial-boundary value problem:

$$
L[u(\mathbf{x}, t)]-\alpha \frac{\partial u}{\partial t}=f(\mathbf{x}, t), \quad \mathbf{x} \in \Omega, t>0
$$




$$
\begin{array}{lc}
u(\mathbf{x}, 0)=\bar{u}(\mathbf{x}), & \mathbf{x} \in \Omega \\
B[u(\mathbf{x}, t)]=g(\mathbf{x}, t), & \mathbf{x} \in \Gamma=\partial \Omega
\end{array}
$$

where $L$ is a time-independent linear differential operator, $B$ is a boundary operator, $u$ denotes the temperature distribution, $f(\mathbf{x}, t)$ represents the heat source term, and $\alpha$ is a coefficient. The domain $\Omega$ is simply connected and the boundary $\Gamma$ is a simple closed boundary curve. In addition, $\bar{u}(\mathbf{x})$ is a given initial temperature in the domain and $g(\mathbf{x}, t)$ gives the boundary value. Assume that the boundary $\Gamma$ consists of three parts $\Gamma=\left(\Gamma_{u} \cup \Gamma_{q} \cup \Gamma_{0}\right)$ and the boundary conditions are given as

$$
\begin{array}{ll}
u(\mathbf{x}, t)=u_{0}(\mathbf{x}, t) & \mathbf{x} \in \Gamma_{u} \\
q(\mathbf{x}, t)=u_{i}(\mathbf{x}, t) n_{i}=q_{0}(\mathbf{x}, t) & \mathbf{x} \in \Gamma_{q}
\end{array}
$$

or

$$
\begin{array}{ll}
B=1, g(\mathbf{x}, t)=u_{0}(\mathbf{x}, t) & \mathbf{x} \in \Gamma_{u} \\
B=\partial / \partial x_{i} n_{i}, g(\mathbf{x}, t)=q_{0}(\mathbf{x}, t) & \mathbf{x} \in \Gamma_{q}
\end{array}
$$

where $n_{i}$ is the unit outward normal at the boundary. The IHCP to be investigated in this paper is to determine the temperature and heat flux on unreachable boundary $\Gamma_{0}$ from given Dirichlet data on $\Gamma_{u}$, Neumann data on $\Gamma_{q}$ and scattered measurement temperature data $u_{T}(\mathbf{x}, t)$ at some points $P_{T}$ either in the domain $\Omega$ or on the Neumann data boundary $\Gamma_{q}$. We first apply Laplace transformation on Eq. (1) to obtain

$$
\begin{array}{ll}
L[\tilde{u}(\mathbf{x}, s)]-s \alpha \tilde{u}(\mathbf{x}, s)=\tilde{f}(\mathbf{x}, s)+\alpha u_{0}(\mathbf{x}), & \mathbf{x} \in \Omega \\
\tilde{u}(\mathbf{x}, s)=\tilde{u}_{0}(\mathbf{x}, s), & \mathbf{x} \in \Gamma_{u} \\
\tilde{q}(\mathbf{x}, s)=\tilde{q}_{0}(\mathbf{x}, s), & \mathbf{x} \in \Gamma_{q} \\
\tilde{u}(\mathbf{x}, s)=\tilde{u}_{T}(\mathbf{x}, s), & \mathbf{x} \in P_{T}
\end{array}
$$

in which $s$ denotes the Laplace transform parameter and

$$
\tilde{F}(\mathbf{x}, s)=\int_{0}^{\infty} e^{-s t} F(\mathbf{x}, t) d t
$$

Based on the idea of radial basis functions, the approximation of a function $R$ can be made by a finite series of radial basis functions $\left\{\varphi_{j}\right\}_{1}^{n}$ through the following interpolation

$$
R(\mathbf{x}) \approx \sum_{i=1}^{n} a_{i} \varphi_{i}(r)
$$


where $r=\left\|\mathbf{x}-\mathbf{x}_{i}\right\|$ is the Euclidean distance, $\left\{\left(x_{i}, y_{i}\right)\right\}_{1}^{n}$ for 2-D or $\left\{\left(x_{i}, y_{i}, z_{i}\right)\right\}_{1}^{n}$ for 3-D is a unisolvent set of interpolation points, and $\left\{a_{i}\right\}_{1}^{n}$ are unknown coefficients to be determined and are the total number of collocation points.

\subsection{Approximation by particular solutions}

By introducing an approximated solution of displacement:

$$
\tilde{u}(\mathbf{x})=\sum_{i=1}^{n} a_{i} \Psi_{i}(r)
$$

where $\left\{\Psi_{i}\right\}_{1}^{n}$ are particular solutions which can be obtained analytically from Eq. (3) by solving

$$
\left(L-\lambda^{2}\right) \Psi_{i}(r)=\varphi_{i}(r), \quad 1 \leq i \leq n
$$

where $\lambda^{2}=s \alpha$ and $\varphi_{i}(r)$ is a set of radial basis functions. If $L=\nabla^{2}$ and $\varphi(r)=r^{2} \ln r$, the particular solution can be expressed as:

$$
\Psi(r)= \begin{cases}-\frac{4}{\lambda^{4}}\left(K_{0}(\lambda r)+\ln r\right)-\frac{r^{2} \ln r}{\lambda^{2}}-\frac{4}{\lambda^{4}}, & r>0 \\ \frac{4}{\lambda^{4}}\left(\gamma+\ln \left(\frac{\lambda}{2}\right)\right)-\frac{4}{\lambda^{4}}, & r=0\end{cases}
$$

where $K_{0}(z)$ is the Bessel function of the second kind of order zero and $\gamma=0.57721566$. Therefore we obtain from Eq. (3) that

$$
\sum_{i=1}^{n} a_{i} \varphi_{i}(r)=\tilde{f}(\mathbf{x}, s)+\alpha u_{0}(\mathbf{x}), \quad \mathbf{x} \in \Omega
$$

for the points in the domain, and

$$
\begin{array}{ll}
\sum_{i=1}^{n} a_{i} \Psi_{i}(r)=\tilde{u}_{0}(\mathbf{x}, s), & \mathbf{x} \in \Gamma_{u} \\
\sum_{i=1}^{n} a_{i} B \Psi_{i, k}(r) n_{k}=\tilde{q}_{(}(\mathbf{x}, s), & \mathbf{x} \in \Gamma_{q} \\
\sum_{i=1}^{n} a_{i} \Psi_{i}(r)=\tilde{u}_{T}(\mathbf{x}, s), & \mathbf{x} \in P_{T}
\end{array}
$$

for the points on the boundary. For numerical implementation, we let $\left\{\mathbf{x}_{i}\right\}_{1}^{n_{i}}$ be the interior collocation points $\left(n_{i}\right),\left\{\mathbf{x}_{i}\right\}_{n_{i}^{+}+1}^{n_{i}+n_{b}}$ be the boundary collocation points $\left(n_{b}\right)$, and $\left\{\mathbf{x}_{i}\right\}_{n_{i}+n_{b}+1}^{n_{i}+n_{b}+m_{u}+m_{q}+m_{T}}$ be the given 
boundary value points $\left(m_{u}+m_{q}\right)$ and scattered temperature measurement points $\left(m_{T}\right)$. Generally we should have $n_{i}+m_{u}+m_{q}+m_{T}=m \geq n=n_{i}+n_{b}$. By the collocation method we obtain from Eqs. (8) and (9)

$$
\begin{array}{ll}
\sum_{i=1}^{n} a_{i} \varphi_{i}\left(r_{k}\right)=\tilde{f}\left(\mathbf{x}_{k}, s\right)+\alpha u_{0}\left(\mathbf{x}_{k}\right), & \mathbf{x} \in \Omega, 1 \leq k \leq n_{i} \\
\sum_{i=1}^{n} a_{i} \Psi_{i}\left(r_{k}\right)=\tilde{u}_{0}\left(\mathbf{x}_{k} s\right), & \mathbf{x} \in \Gamma_{u}, n_{i}+1 \leq k \leq n_{i}+m_{u} \\
\sum_{i=1}^{n} a_{i} \Psi_{i, j}\left(r_{k}\right) n_{j}=\tilde{q}\left(\mathbf{x}_{k}, s\right), & \mathbf{x} \in \Gamma_{q}, n_{i}+m_{u}+1 \leq k \leq n_{i}+m_{u}+m_{q} \\
\sum_{i=1}^{n} a_{i} \Psi_{i}\left(r_{k}\right)=\tilde{u}_{T}\left(\mathbf{x}_{k} s\right), & \mathbf{x} \in P_{T}, n_{i}+m_{u}+m_{q}+1 \leq k \leq n_{i}+m_{u}+m_{q}+m_{T}
\end{array}
$$

If we choose $n=m$, all unknown coefficients $\left\{a_{i}\right\}_{1}^{n}$ can be easily computed by using a standard matrix solver such as Gaussian elimination and $L U$ decomposition. Once the values of $\left\{a_{i}\right\}$ are determined, the approximated solution $\tilde{u}$ can be evaluated from Eq. (6). Therefore, the unknown temperature and flux density can be determined on the unreachable boundary $\Gamma_{0}$.

\subsection{Approximation by semi-particular solutions}

Similar to Section 2.1, we assume an interpolation of the displacement in Eq. (3) by the particular solutions $\left\{\Psi_{i}\right\}_{1}^{n}$ which can be obtained analytically by solving

$$
L \Psi_{i}(r)=\varphi_{i}(r), \quad 1 \leq i \leq n
$$

where $\varphi_{i}(r)$ is a radial basis function and the particular solution $\Psi_{i}(r)$ is determined by solving the above Eq. (12). Substituting Eq. (12) into Eq. (3) gives

$$
\sum_{i=1}^{n}\left(\varphi_{i}(r)-s \alpha \Psi_{i}\right) a_{i}=\tilde{f}(\mathbf{x}, s)+\alpha u_{0}(\mathbf{x}), \quad \quad \mathbf{x} \in \Omega
$$

in the domain with the same boundary conditions given in Eq. (10). By collocation method we have

$$
\sum_{i=1}^{n}\left(\varphi_{i}\left(r_{k}\right)-s \alpha \Psi_{i}\left(r_{k}\right)\right) a_{i}=\tilde{f}\left(\mathbf{x}_{k}, s\right)+\alpha u_{0}\left(\mathbf{x}_{k}\right), \quad \mathbf{x} \in \Omega, 1 \leq k \leq m_{i}
$$


with boundary equations $(11 \mathrm{a}, 11 \mathrm{~b}, 11 \mathrm{c})$ and $(11 \mathrm{~d})$. The approximated solution $\tilde{u}$ can then be evaluated from Eq. (6) when $n=m$. For instance, if $L=\nabla^{2}$ we have the particular solutions

$$
\Psi(r)=r^{4}(2 \ln r-1) / 32 \text { for } \varphi(r)=r^{2} \ln r
$$

and

$$
\Psi(r)=\left(4 b^{2}+r^{2}\right) \sqrt{r^{2}+\vec{b}^{2}} / 9-b^{3} \ln \left(b+\sqrt{r^{2}+\vec{b}^{2}}\right) / 3 \text { for } \varphi(r)=\sqrt{r^{2}+\vec{b}^{2}}
$$

where $b$ is a free parameter.

\section{Inversion of Laplace Transformation}

\subsection{Singular value decomposition of a square matrix}

Using the Laplace transform, we can rewrite the system of algebraic equations into a matrix form with unknown quantities on the left-hand side and prescribed quantities on the right-hand side as

$$
\mathbf{A}_{m \times n} \mathbf{X}_{n}=\mathbf{Y}_{m}
$$

Since the original IHCP is ill-posed, the ill-conditioning of the matrix $\mathbf{A}$ in Eq. (17) still persists. It means that numerical results are sensitive to the noise of the right-hand side and the number of nodes used in the meshless approximation. The worst case will occur if no temperature data are given at internal points and the Dirichlet and Neumann boundary conditions are prescribed at the same points [17]. If $n=m$, the square matrix $\mathbf{A}$ can be decomposed according to the singular value decomposition (SVD) [25] and its inverse can be written as

$$
\mathbf{A}^{-1}=\mathbf{V} \cdot\left[\operatorname{diag}\left(1 / w_{j}\right)\right] \cdot \mathbf{U}^{T}
$$

where $\mathbf{U}, \mathbf{V}$, and $\mathbf{W}$ are all square matrices of the same size, $\mathbf{W}=\left[w_{j}\right], \mathbf{U}$ and $\mathbf{V}$ are orthogonal, i.e.,

$$
\begin{array}{ll}
\sum_{i=1}^{n} U_{i k} U_{i j}=\delta_{k j} & 1 \leq k \leq n \\
& 1 \leq j \leq n \\
\sum_{i=1}^{n} V_{i k} V_{i j}=\delta_{k j} & 1 \leq k \leq n \\
& 1 \leq j \leq n
\end{array}
$$


so that their inverses are equal to their transpose. The matrix $\mathbf{W}$ is diagonal and its inverse is the diagonal matrix whose elements are reciprocals of the elements $w_{j}$. Based on the SVD, the solution for the ill-posed linear equations Eq. (17) is given by

$$
\mathbf{X}=\mathbf{V} \cdot\left[\operatorname{diag}\left(1 / \mathrm{w}_{\mathrm{j}}\right)\right] \cdot\left(\mathbf{U}^{\mathrm{T}} \cdot \mathbf{Y}\right)
$$

We note here that in the case when the system Eq. (17) is over-determined, i.e., $m>n$ the least-square technique should be used to solve Eq. (17) before using SVD. Now, applying the transpose of matrix A to both sides of Eq. (17) gives

$$
\left[\mathbf{A}^{\mathrm{T}}\right]_{\mathrm{n} \times \mathrm{m}}[\mathbf{A}]_{\mathrm{m} \times \mathrm{n}}\{\mathbf{X}\}_{\mathrm{n}}=\left[\mathbf{A}^{\mathrm{T}}\right]_{\mathrm{n} \times \mathrm{m}}\{\mathbf{Y}\}_{\mathrm{m}}
$$

which gives the solution for the ill-conditioning matrix equation system.

\subsection{Durbin inversion of the Laplace transform}

We first select a total number of $(L+1)$ samples in the transformation space $s_{k}, k=0,1,2, \ldots$ ., $L$, as complex variables. The transformed variables are evaluated for these specified transform parameters respectively. The unknown solution in the time domain can then be determined by using the Laplace inversion technique. There are many inversion methods available for numerical computing of the inverse Laplace transform. For instance, the sophisticated Stehfest algorithm [27] proposed by Sladek et al. [17] for solving IHCP is one such method. In this paper we adopt the method proposed by Durbin [26] which had been applied by Wen et al. [28] for solving elasticity wave propagation problems in two- and three-dimensional spaces. The formulae of the Durbin inversion method is written as

$$
\mathrm{F}(\mathrm{t})=\frac{2 \mathrm{e}^{\omega \mathrm{tt}}}{\mathrm{T}}\left[-\frac{1}{2} \tilde{\mathrm{F}}\left(\mathrm{s}_{0}\right)+\sum_{\mathrm{k}=0}^{\mathrm{L}} \operatorname{Re}\left\{\tilde{\mathrm{F}}\left(\mathrm{s}_{\mathrm{k}}\right) \mathrm{e}^{2 \mathrm{k} \pi \mathrm{i} / \mathrm{T}}\right\}\right]
$$

where $\tilde{F}\left(s_{k}\right)$ denotes the transformed variable in the Laplace domain and $i=\sqrt{-1}$. The parameter of the Laplace transform is taken to $s_{k}=\omega+2 k \pi i / T$. The selection of two free parameters $\omega$ and $T$ has little effect on the accuracy of the inversion. By a large number of numerical tests, we can conclude that the Durbin inversion method is stable and convergent for a large range selection of free parameters $\omega$ and $T$, particularly for the solid dynamic mechanics.

\section{Numerical Verification}

For simplicity, we assume that the heat conduction coefficient $\alpha=1$. For numerical error estimation, we define the relative average error by the following formula

$$
\mathrm{MEU}=\frac{1}{\mathrm{Nu}_{\max }^{*}} \sum_{i=1}^{\mathrm{N}}\left|\mathrm{u}\left(\mathbf{x}, \mathrm{t}_{\mathrm{i}}\right)-\mathrm{u}^{*}\left(\mathbf{x}, \mathrm{t}_{\mathrm{i}}\right)\right|
$$




$$
M E Q=\frac{1}{N q_{\max }^{*}} \sum_{i=1}^{N}\left|q\left(\mathbf{x}, t_{i}\right)-q^{*}\left(\mathbf{x}, t_{i}\right)\right|
$$

where $N$ is the number of testing nodes located randomly within the domain. In our following computations, we choose $N$ to be 101. Let $u^{*}$ and $q^{*}$ denote the analytical solutions at time $t_{i}$ in the region $\left[0, t_{\max }\right]$. In the cases when the measurement data contain some random noises, we use noisy data input as $u_{T}(\mathbf{x}, t)=u_{T}^{*}(\mathbf{x}, t)[1+\sigma \cdot \operatorname{rand}(\mathbf{x})]$, where $u_{T}^{*}(\mathbf{x}, t)$ is the exact data and $\operatorname{rand}(\mathbf{x})$ is a random number between $[-1,1]$. The magnitude of $\sigma$ denotes the error level.

\subsection{A rectangular plate with one unknown boundary condition}

Consider a square plate with zero initial temperature and zero heat source $f(\mathbf{x}, t)=0$ as shown in Fig. 1. The boundary conditions are described as

$$
\begin{gathered}
q(\mathbf{x}, t)=0 \\
q(\mathbf{x}, t)=0 \\
q(\mathbf{x}, t)=\left\{\begin{array}{cl}
0 & t \in[0,1 / 4] \cup[3 / 4,1] \\
4 t-1 & t \in[1 / 4,1 / 2] \\
3-4 t & t \in[1 / 2,3 / 4]
\end{array} \quad x=0\right.
\end{gathered}
$$

whose analytical solution of temperature can be derived as [5]

$$
u^{*}(\mathbf{x}, t)=\int_{0}^{t} q(\tau) d \tau+2 \sum_{k=1}^{\infty} \cos k \pi x \int_{0}^{t} q(\tau) e^{-k^{2} \pi^{2}(t-\tau)} d \tau
$$

and hence the temperature and flux in the Laplace domain are given by

$$
\tilde{u}^{*}(\mathbf{x}, s)=\left(\frac{1}{s}+2 \sum_{k=1}^{\infty} \frac{\cos k \pi x}{s+k^{2} \pi^{2}}\right) \widetilde{q}(s), \widetilde{q}_{x}^{*}(\mathbf{x}, s)=-2 \sum_{k=1}^{\infty} \frac{k \pi \sin k \pi x}{s+k^{2} \pi^{2}} \widetilde{q}(s), \widetilde{q}_{y}^{*}(\mathbf{x}, s)=0
$$

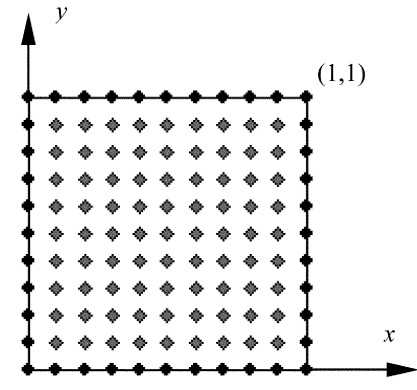

(a)

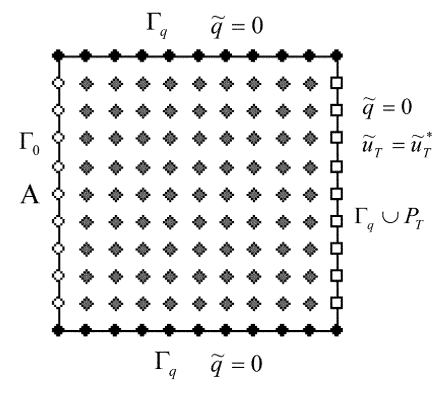

(b)

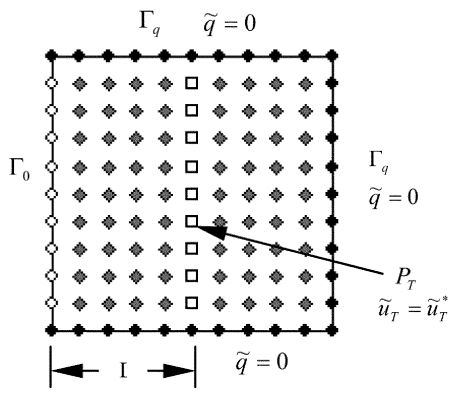

(c)

Fig. 1. Distribution of collocation points: (a) internal and boundary points; (b) boundary conditions for inverse heat conduction problems, where F domain point, ! unknown value boundary, A flux boundary, " Neumann and Dirichlet boundary; (c) $P_{T}$ in domain, where D indicates the gap. 
Table 1. Relative Average Error with Varying Noise Inputs

\begin{tabular}{|c|c|c|c|c|}
\hline$\sigma$ & 0.0 & 0.01 & 0.1 & 1.0 \\
\hline$M E U$ & 0.0292 & 0.0286 & 0.0239 & 0.0751 \\
\hline$M E Q$ & 0.0445 & 0.0447 & 0.0466 & 0.1106 \\
\hline
\end{tabular}

where

$$
\tilde{q}(s)=\frac{4}{s^{2}}\left[e^{-3 s / 4}-2 e^{-s / 2}+e^{-s / 4}\right]
$$

The interior and boundary points are selected as shown in Fig. 1 with $n_{i}=9 \times 9=81$ internal points and $n_{b}=40$ boundary points $\left(n=n_{i}+n_{b}\right)$, i.e., $m_{u}=0, m_{q}=11+11+9=31$ where the scattered temperature measurement points are collected on the right-hand side of the square plate with $m_{T}=9$. The unreachable boundary $\Gamma_{0}$ is specified on $x=0$. The particular solutions to be used are given in Eqs. (8), (9), and (10). Numerical tests show that these two free parameters should be selected as $\omega$

$=5 / T$ and $T=2$ are chosen in the Durbin inversion method of the Laplace transform. The number of sample points in the Laplace transform domain is selected as $L=100$. In this case, as $n=m=121$, the SVD can be applied directly to solve the linear system of equations for the solutions of the temperate and the heat flux. For illustration, the temperature $u(t)$ and the heat flux $q_{x}(t)=\partial u / \partial x$ at the central point on the edge A $(0,0.5)$ with noisy data $\sigma \cdot \operatorname{rand}(\mathbf{x})$ are displayed in Fig. 2. At point A, the analytical solution $u_{\max }^{*}=0.4359$ when $t=0.59$ and $q_{\max }^{*}=1$ when $t=0.5$, respectively. The numerical results shown in Table 1 indicate that the proposed scheme is accurate and stable in
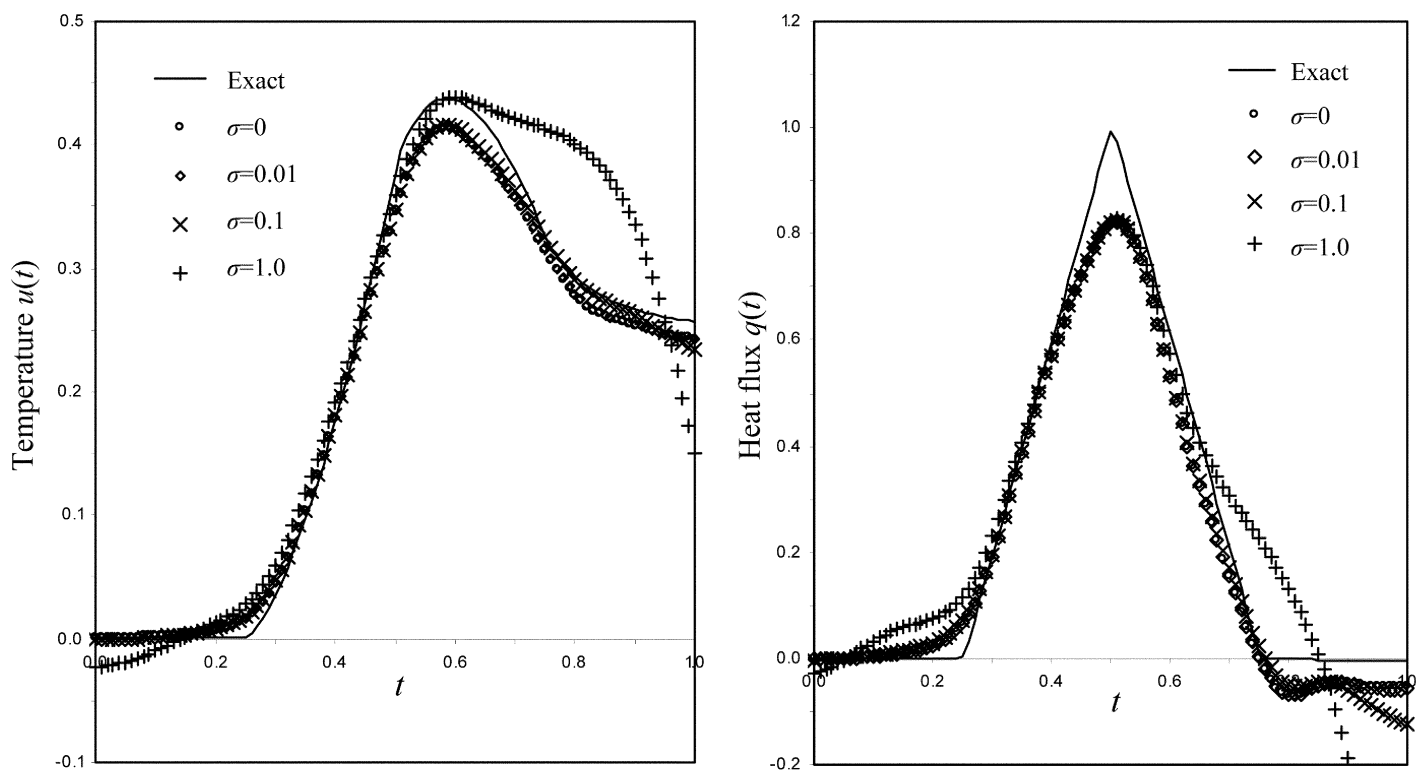

Fig. 2. The boundary temperature $u(t)$ and heat flux $q(t)$ on point A with noisy data $\sigma \operatorname{rand}(\mathbf{x})$ corresponding to the boundary condition (b). The solid line represents the exact solution. 

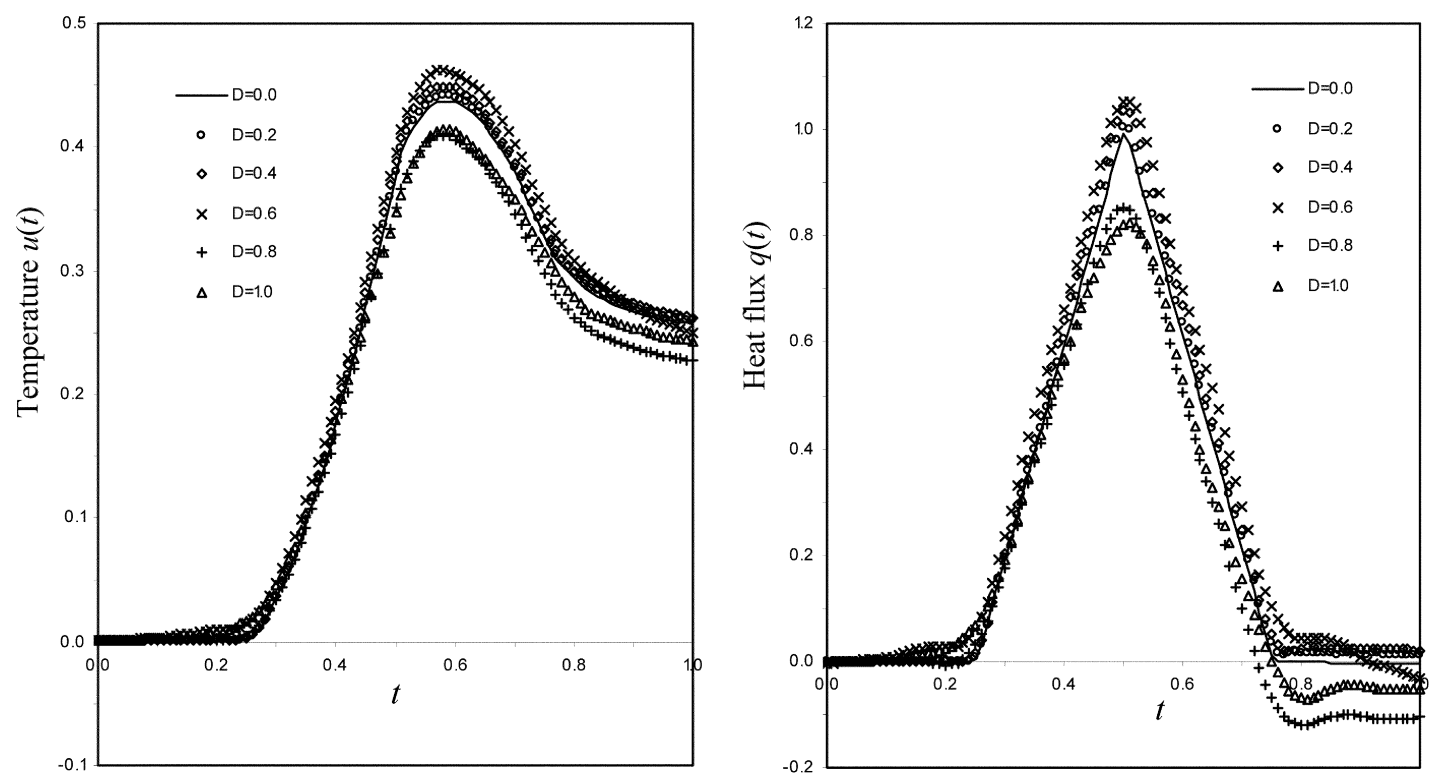

Fig. 3. The boundary temperature $u(t)$ and heat flux $q(t)$ on point A corresponding to the boundary condition (c). The solid line represents the exact solution.

comparing with most of the existing numerical methods. Apparently in the cases when $\sigma<0.1$, the numerical approximations are all close to the analytical solution. It is noted here that it is unusual to obtain such an accurate result for temperature with large noise. Furthermore, we change the scattered temperature data points from the boundary to the interior domain as shown in Fig. 1(c). Figure 3 shows the variation of the temperature $u(t)$ and heat flux $q(t)$ on the boundary point A at time $t$ with different gaps of D. Note that it becomes a well-posed problem without an unreachable boundary if $\mathrm{D}=0$. From Fig. 3 it can be observed that the more accurate solution could be obtained with a smaller gap. To illustrate the stability of the proposed method for solving IHCP, the relative average errors of temperature and heat flux at point A with different gaps are shown in Table 2.

In addition, we observed the convergence with respect to the distribution density of nodes in the domain and on the boundary. The interior and boundary points are selected as $n_{i}=15 \times 15=255$ internal points and boundary points $n_{b}=64$. The numerical results show that the differences between these two node distributions are very small. Regarding the choice of the optimal truncation number associated with the SVD, we used the source code in Ref. 25 directly and set the

threshold for singular values allowed to be nonzero, i.e., $W M I N=W M A X^{*} 1.0 E-6$.

Table 2. Relative Average Error with Different Gaps D

\begin{tabular}{|c|c|c|c|c|c|c|}
\hline $\mathrm{D}$ & 0.0 & 0.2 & 0.4 & 0.6 & 0.8 & 1.0 \\
\hline$M E U$ & 0.0023 & 0.0055 & 0.0138 & 0.0302 & 0.0390 & 0.0292 \\
\hline$M E Q$ & 0.0054 & 0.0122 & 0.0274 & 0.0517 & 0.0618 & 0.0445 \\
\hline
\end{tabular}




\subsection{A ring with unreachable boundary on inner circle}

Consider a quarter of a ring as shown in Fig. 4. The differential heat conduction equation in a polar coordinate system can be written as

$$
\frac{\partial^{2} \tilde{u}}{\partial r^{2}}+\frac{1}{r}-\partial \tilde{u}+\frac{1}{r^{2}}-\frac{\partial^{2} \tilde{u}}{\partial \theta^{2}}-\alpha s \tilde{u}=\tilde{f}(r, \theta, s)+\alpha u_{0}(r, \theta)
$$

For axial symmetric problems, the general solution of Eq. (28) is obtained by

$$
\begin{gathered}
\tilde{u}(r, s)=A I_{0}(\sqrt{\alpha s} r)+B K_{0}(\sqrt{\alpha s} r) \\
\tilde{q}(r, s)=\sqrt{\alpha s}\left[A I_{1}(\sqrt{\alpha s} r)-B K_{1}(\sqrt{\alpha s} r)\right]
\end{gathered}
$$

where the coefficients $A$ and $B$ are to be determined by using the boundary conditions. The Neumann boundary conditions are given on the inner and outer circles as

$$
\begin{array}{ll}
\tilde{q}(r, s)=\tilde{q}_{a}(s) & r=a \\
\tilde{q}(r, s)=\tilde{q}_{b}(s) & r=b
\end{array}
$$

Therefore, we obtain the analytical solution in the Laplace domain as

$$
\begin{aligned}
& \tilde{u}^{*}(r, s)=\frac{\left[\widetilde{q}_{a} K_{1}(\sqrt{\alpha s} b)-\widetilde{q}_{b} K_{1}(\sqrt{\alpha s} a)\right] I_{0}(\sqrt{\alpha s} r)-\left[\widetilde{q}_{b} I_{1}(\sqrt{\alpha s} a)-\widetilde{q}_{a} I_{1}(\sqrt{\alpha s} b)\right] K_{0}(\sqrt{\alpha s} r)}{\sqrt{\alpha s}\left[I_{1}(\sqrt{\alpha s} a) K_{1}(\sqrt{\alpha s} b)-I_{1}(\sqrt{\alpha s} b) K_{1}(\sqrt{\alpha s} a)\right]} \\
& \widetilde{q}^{*}(r, s)=\frac{\left[\widetilde{q}_{a} K_{1}(\sqrt{\alpha s} b)-\widetilde{q}_{b} K_{1}(\sqrt{\alpha s} a)\right] I_{1}(\sqrt{\alpha s} r)+\left[\widetilde{q}_{b} I_{1}(\sqrt{\alpha s} a)-\widetilde{q}_{a} I_{1}(\sqrt{\alpha s} b)\right] K_{1}(\sqrt{\alpha s} r)}{\left[I_{1}(\sqrt{\alpha s} a) K_{1}(\sqrt{\alpha s} b)-I_{1}(\sqrt{\alpha s} b) K_{1}(\sqrt{\alpha s} a)\right]}
\end{aligned}
$$

Assuming that the boundary conditions can be described in $x, y$ coordinate system in time domain as

$$
\begin{array}{ccc}
q^{*}(t)=0 & x=0 & \\
q^{*}(t)=0 & y=0 & \\
q^{*}(t)=0 & r=a & \\
q^{*}(t)=H(t-1)-H(t-2) & & r=b
\end{array}
$$

where $H(t)$ denotes the Heaviside function. Thus, the boundary conditions in the transform domain are

$$
\begin{array}{ll}
\tilde{q}^{*}(s)=0 & x=0 \\
\tilde{q}^{*}(s)=0 & y=0 \\
\tilde{q}^{*}(s)=0 & r=a
\end{array}
$$




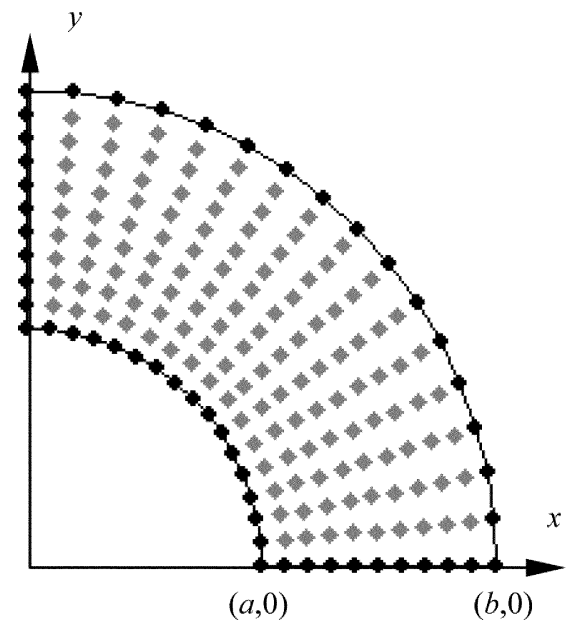

(a)

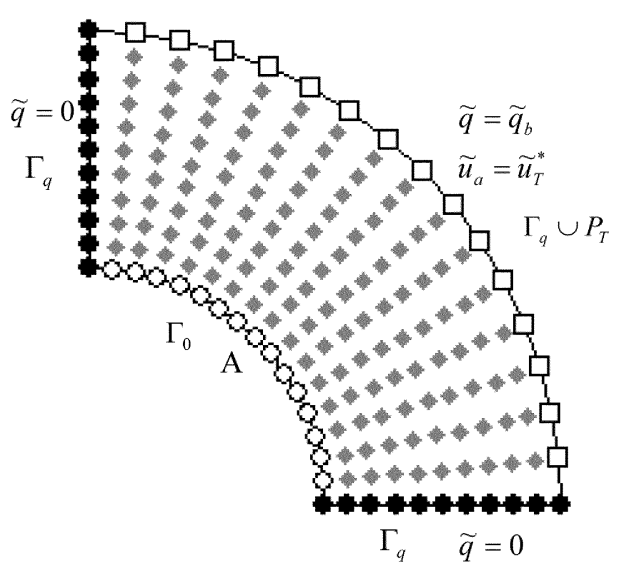

(b)

Fig. 4. Distribution of collocation points in a quarter of ring: (a) internal and boundary points; (b) boundary conditions for inverse heat conduction problems, where F domain point, ! unknown value boundary, A flux boundary, " Neumann and Dirichlet boundary $P_{T}$.

$$
\tilde{q}^{*}(s)=\frac{1}{s}\left[e^{-s}-e^{-2 s}\right] \quad r=b
$$

Figure 4 shows the distribution of interior and boundary points, where $n_{i}=9 \times 15=135$ and $n_{b}=$ 48 , and $m_{u}=0, m_{q}=11+15+11=37$. The measurement points are collected on the outer circle $r$ $=b$ with the number of points $m_{T}=15$. The temperature and flux on the unreachable boundary $\Gamma_{0}$ are specified at the inner circle $r=a$. The semi-particular solutions to be used are given by Eqs. (15) and (16). In this computation, we fix the parameters $\alpha=1, a=1$, and $b=2$, respectively. The two free parameters are chosen to be $\omega=5 / T$ and $T=10$ in the Durbin inversion method of the Laplace

transform. The total number of sample points in the Laplace transform domain is selected as $L=100$. As $n=m=135+37+15=187$, the SVD can be applied directly to obtain the solutions. The normalized temperature $u$ and the flux $q=\partial u / \partial r$ at point A $(\sqrt{2} / 2, \sqrt{2} / 2)$ of the interior circle with noisy data $\sigma$ $\cdot \operatorname{rand}(\mathbf{x})$ are shown in Fig. 5. It can be observed that there is very little difference between the analytical solution and the numerical approximation of temperature when $\sigma<0.01$. The relative average error of the temperature increases only to $15 \%$ for the noise magnitude when $\sigma=0.1$.

\subsection{A rectangular plate with a circular hole}

Consider a square plate containing a circular hole with zero initial temperature as shown in Fig. 6. Let the exact solution of temperature be

$$
u^{*}(r, t)=1-e^{-1}+t r^{2} e^{-t}
$$

and heat flux 

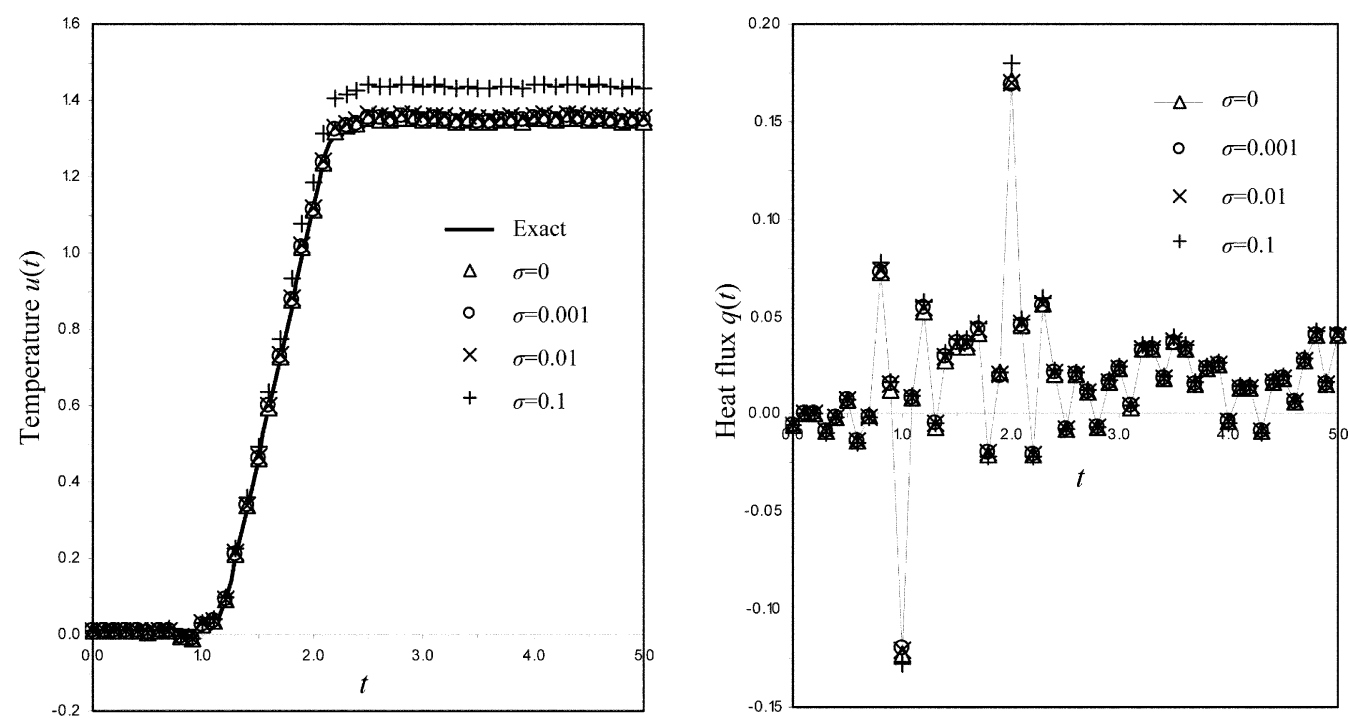

Fig. 5. The boundary temperature $u(t)$ and heat flux $q(t)$ at point A corresponding to the boundary condition (b).

$$
q_{x}^{*}(r, t)=2 x t e^{-t}, q_{y}^{*}(r, t)=2 y t e^{-t}
$$

with the source term

$$
f(r, t)=\left[4 t-(1-t) r^{2}-1\right] e^{-t}
$$

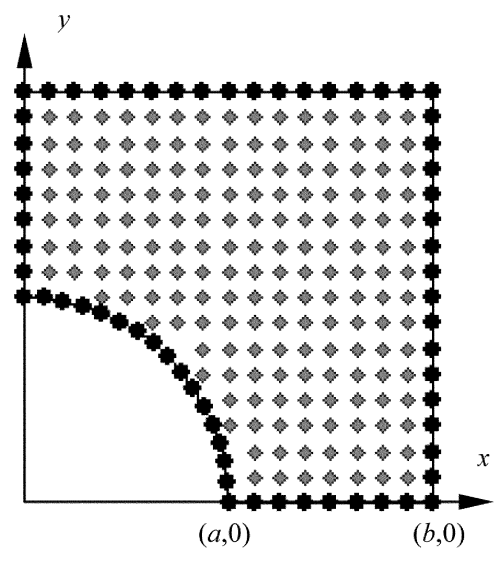

(a)

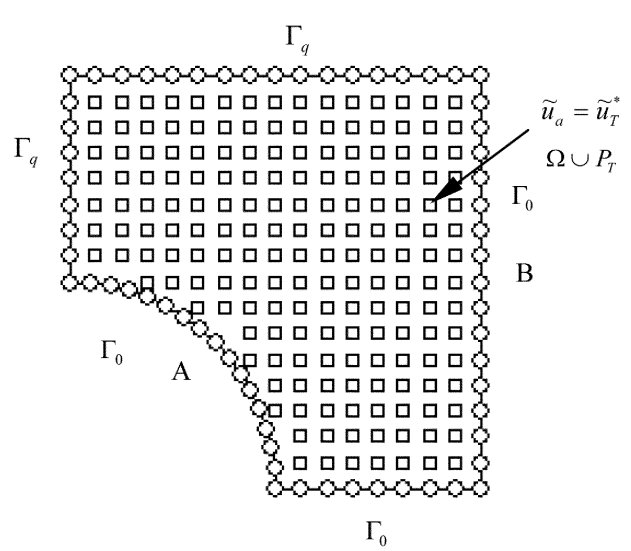

(b)

Fig. 6. Distribution of collocation points in a quarter of ring: (a) internal and boundary points, where F domain point and A boundary; (b) boundary conditions for inverse heat conduction problems, where ! unknown value boundary, " Dirichlet point $P_{T}$ in domain. 

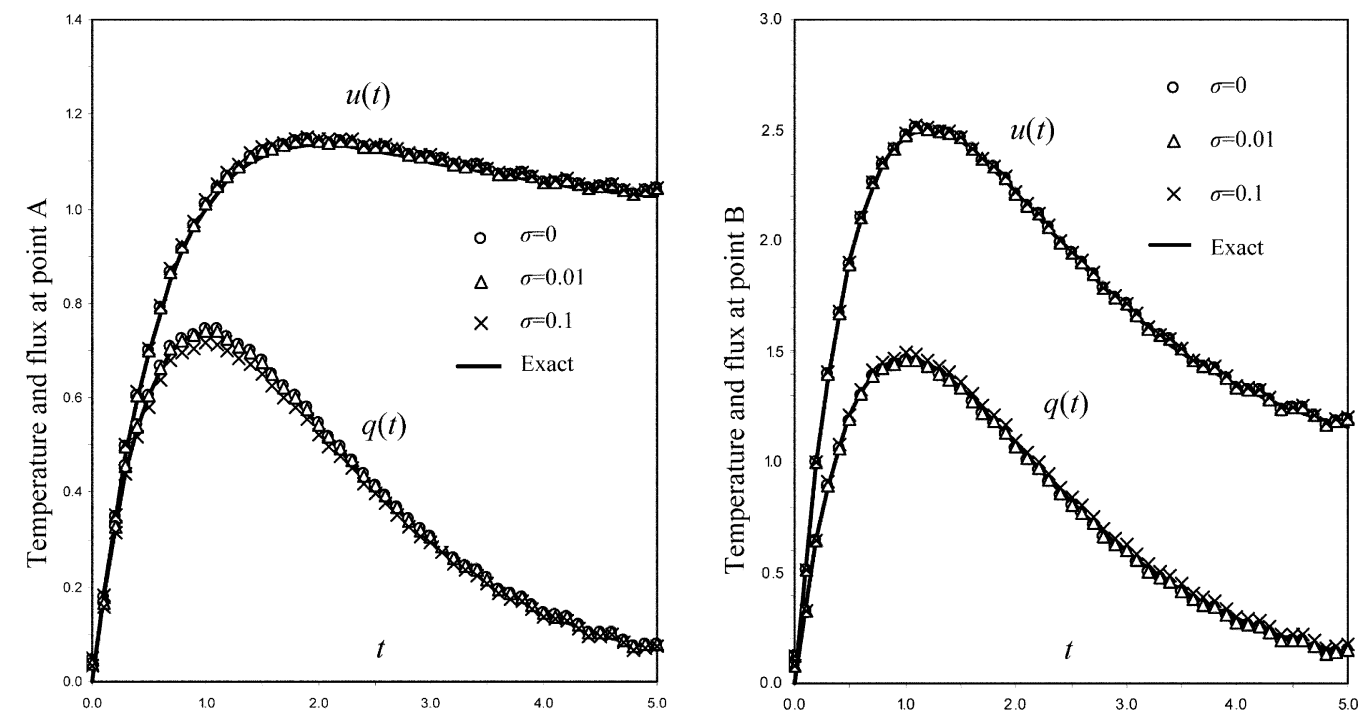

Fig. 7. The boundary temperature $u(t)$ and heat flux $q(t)$ at point A corresponding to the boundary condition (b).

Then the exact solution in the Laplace domain is given by

$$
\tilde{u}^{*}(r, s)=\frac{r^{2}}{(s+1)^{2}}+\frac{1}{s(s+1)}, \tilde{q}_{x}^{*}(r, s)=\frac{2 x}{(s+1)^{2}}, \tilde{q}_{y}^{*}(r, s)=\frac{2 y}{(s+1)^{2}}
$$

In this example, the semi-particular solutions to be used are given by Eqs. (15) and (16). The IHCP to be investigated is to determine the temperature and heat flux on the unreachable boundary from given scattered measurement data at all interior points. The interior and boundary collocation points are distributed with $n_{i}=177$ and $n_{b}=64$. We assume that the whole boundary is unreachable boundary $\Gamma_{0}$, i.e., $m_{u}=m_{q}=0$, and the scattered temperature measurement are collected in the interior domain at each collocation points, that is $m_{T}=n_{i}=177$. In this case, the total number of collocation points is $n=n_{i}+n_{b}=241$ and the number of linear equations is $m=n_{i}+m_{T}=354$. Due to $m>n$, the least-square technique is used first before applying the SVD. The parameters are selected by $\alpha=1, a=1$, and $b$ $=2$, respectively. The two free parameters are $\omega=5 / T$ and $T=10$ in the Durbin inversion method of the Laplace transform. The temperature $u$ and the heat fluxes $q=\partial u / \partial r$ at central point $\mathrm{A}(\sqrt{2} / 2, \sqrt{2} / 2)$ of the plate with noisy data $\sigma \cdot \operatorname{rand}(\mathbf{x})$ are displayed in Fig. 7 , where the analytical solutions are plotted in solid lines for comparison. Apparently the numerical results using the proposed method are efficient and satisfactory even for large measurement noises.

\section{Conclusions}

In the past, the availability of fundamental solutions and particular solutions are equally important for solving inhomogeneous problems. In this paper, we propose that only the particular solution is required without the need of a fundamental solution for solving these inhomogeneous problems. The approach based on using the particular solution as the radial bases function approxi- 
mation together with using the Laplace transform technique for time variable is presented for solving the transient heat conduction inverse problems. The domain and boundary formulations can be easily implemented and the coefficients of the particular solutions can be obtained by solving the linear system of equations. Singular value decomposition (SVD) is applied to solve the ill-conditioned linear system of algebraic equations obtained from the differential equation, boundary conditions, and scattered temperature measurement data. Stable and accurate results for inverse heat conduction problems are obtained even with considerable large noise contained in the scattered input measurement data. We believe that the proposed method of using particular/semi-particular solutions provides an efficient scheme for solving these kinds of inverse heat conduction problems.

\section{Acknowledgments}

The work described in this paper was partially supported by a grant from the Research Grant Council of the Hong Kong Special Administrative Region, China (Project No. CityU 101209).

\section{Literature Cited}

1. Jonas P, Louis AK. Approximate inverse for a one-dimensional inverse heat conduction problem. Inverse Problems 2000;16:175-185.

2. Lesnic D, Elliott L. The decomposition approach to inverse heat conduction. J Math Anal Appl 1999;232:82-98.

3. Liu J. A stability analysis on Beck's procedure for inverse heat conduction problem. J Comput Phys 1996;123:65-173.

4. Shen SY. A numerical study of inverse heat conduction problems. Comput Math Appl 1999;38:173-188.

5. Hon YC, Wei T. A fundamental solution method for inverse heat conduction problem. Eng Anal Bound Elem 2004;28:489-495.

6. Chantasiriwan S. An algorithm for solving multidimensional inverse heat conduction problem. Int Comm Heat Mass Transf 2001;44:3823-3832.

7. Kurpisz K, Nowak AJ. BEM approach to inverse heat conduction problems. Eng Anal Bound Elem 1992;10:291-297.

8. Guo L, Murio DA. A modified space-marching finite-difference algorithm for the two-dimensional inverse heat conduction problem with slab symmetry. Inverse Probl 1991;7:247-259.

9. Khalidy NA. A general space marching algorithm for the solution of two-dimensional boundary inverse heat conduction problems. Numer Heat Transf Part B 1998;34:339-360.

10. Hsu TR, Sun NS, Chen GG, Gong ZL. Finite element formulation for two-dimensional inverse heat conduction analysis. ASME J Heat Transf 1992;114:553-557.

11. Reinhardt HJ. A numerical method for the solution of two-dimensional inverse heat conduction problems. Int J Num Meth Eng 1991;32:363-383.

12. Hon YC, Wei T. The method of fundamental solutions for solving multidimensional inverse heat conduction problems. CMES-Comp Model Eng Sci 2005;7:119-132.

13. Fairweather G, Karageorghis A. The method of fundamental solution for elliptic boundary value problems. Adv Computat Math 1998;9:69-95.

14. Golberg MA, Chen CS. The method of fundamental solutions for potential, Helmholtz and diffusion problems. In: Boundary integral methods-Numerical and mathematical aspects, Golberg MA (ed.), Computational Mechanics Publications: Southampton; 1998.

15. Cho HA, Golberg MA. Trefftz methods for time-dependent partial differential equations. CMC 2004;1:1-37. 
16. Atluri SN. The meshless method (MLPG) for domain and BIE discretizations. Tech Science Press: Forsyth GA; 2004.

17. Sladek J, Sladek V, Hon YC. Inverse heat conduction problems by meshless local PetrovGalerkin method. Engineering Analysis with Boundary Elements 2006;30:650-661.

18. Belytschko T, Lu YY, Gu L. Element-free Galerkin method. Int J Numerical Meth Eng 1994;37:229-256.

19. Golberg MA, Chen CS. A mesh free method for solving nonlinear reaction-diffusion equations. Comp Modeling Eng Sci 2001;2:87-95.

20. Ingber M, Chen CS, Tanski JA. A mesh free approach using radial basis functions and parallel domain decomposition for solving three dimensional diffusion equations. Int J Numer Methods Eng 2004;60:2183-2201.

21. Reutskiy S, Chen CS, Tian H. A boundary meshless method using Chebyshev interpolation and trigonometric basis function for solving heat conduction problems. Int J Numer Methods Eng 2008;74:1621-1644.

22. Cheng AHD. Particular solutions of Laplacian, Helmholtz-type and polyharmonic operators involving higher order radial basis functions. Eng Anal Boundary Elements 2000;24:531-538.

23. Li J. A radial basis meshless method for solving inverse boundary value problems. Commun Numer Meth Eng 2004;20:51-60.

24. Golub GH, Van Loan VF. Matrix computations. The John Hopkins University Press; 1996.

25. Press WH, Flannery BP, Teukolsky SA, Vetterling WT. Numerical recipes in FORTRAN. Cambridge University Press; 1992.

26. Durbin F. Numerical inversion of Laplace transforms: an efficient improvement to Dubner and Abate's method. Comput J 1975;17:371-376.

27. Stehfest H. Algorithm 368: numerical inversion of the Laplace transform. Comm ACM 1970;13:47-49.

28. Wen PH, Aliabadi MH, Rooke DP. The influence of elastic waves on dynamic stress intensity factors (three dimensional problem). Arch Appl Mech 1996;66(6):384-385. 